\title{
Odontogenic abscess with severe and fast evolution in a young and healthy patient
}

Bruna da Fonseca Wastner ${ }^{1}$, Salmo Cortiglio ${ }^{1}$, Joana Letícia Vendruscolo ${ }^{2}$, José Luis Dissenha ${ }^{3}$

Deep cervical abscesses are infections of the different spaces on the the neck that may have a mild or severe outcome and requires fast and assertive approach. The most usual cases of severe infections are associates with comorbidities and older patients, but sometimes, in rare cases, it may affect the younger and healthier. Aim: The purpose of this is to communicate shortly a case of a severe cervical abscess with fast evolution in a 28 years old patient, to highlight the possibility of this outcome even in unexpected cases.

Key-words: Abscess. Infection. Neck.

Corresponding author:

Bruna da Fonseca Wastner Rua Guilherme Tragante, 377,

Tarumã, Curitiba, Paraná, Brazil, 82800-090.

Email: bru.wastner@hotmail.com

Received: December 062017

Accepted: May 11, 2018 


\section{Introduction}

Deep cervical abscesses are defined by the presence of pus in regions of the head and neck, and can reach separately or together the spaces: retropharyngeal, peritonsillar, masseteric, submandibular, parapharyngeal, pterygopalatine and parotid, among others. In some cases, especially when the treatment is not adequate, they can progress both superior and inferior, increasing the risk of death. A rapid and accurate approach is required in order to avoid complications such as airway obstruction, necrotizing fasciitis, jugular vein thrombosis, aspiration pneumonia and carotid artery thrombosis (1). It is observed that even with the use of broad-spectrum antimicrobials and surgical drainage, severe complications may occur, mainly, but not exclusively, in patients with comorbidities (2). The literature shows that airway obstruction forced emergency tracheostomy in $7.9 \%$ of the cases and in those individuals with retropharyngeal spaces and mouth floor, $75 \%$ presented this need (3). The trismus due to the involvement of the masticatory muscles may also be a predisposing factor, even if there is no respiratory distress. Mortality rates in these cases are described as close to $2 \%(2,3)$.

\section{Case report}

Our patient was treated in the emergency room of the Municipal Hospital and Maternity São José dos Pinhais, Paraná, Brazil, with an odontogenic abscess associated with the left lower molars. Intraoral exam showed pericoronitis in the third left molar and a carious lesion in the second left molar. Despite this, patient had a good oral hygiene and absence of periodontal disease. The patient presented hyperthermia and edema in the left submandibular region without a fluctuation point, with dysphagia and dyspnea. He referred that started to feel pain in the region in the day before and, when he woke up on the next day, the swelling was present. He was classified as ASA I, have no comorbidities and all serologies came negative. Initially, empiric intravenous antibiotic therapy was used with cefazolin and metronidazole, but the patient evolved with increased edema and worsened general condition. It was surgically approached through extraoral drainage of two localized colletions, one submandibular and one sublingual. After a few hours, the patient got worse, presenting cervical erythema extending to the clavicle. At this time, the triple antimicrobial regimen was instituted, with the addition of gentamicin, thinking of a more severe infection with gram negative aerobes. However, a few hours later, the erythema reached the lower sternal region and the patient complained of dyspnea and chest pressure. Computed tomography of the neck and thorax showed a purulent collection medial to the left sternocleidomastoid muscle, without compromising the mediastinum and a purulent collection in the retropharyngeal space and on the deep neck space, compromising the airway (Figures 1 and 2). The patient underwent a new surgical drainage. In conjunction with the thoracic surgeon, cervicotomy was performed to access the collection medial to the left sternocleidomastoid muscle and abundant irrigation with a $0.9 \%$ sodium chloride heated solution, maintaining two Penrose drains. The wound was left open for healing by second intention (Figure 3). In the same surgical procedure, the infected tooth was extracted. Culture of the secretion collected in the trans-operative revealed 


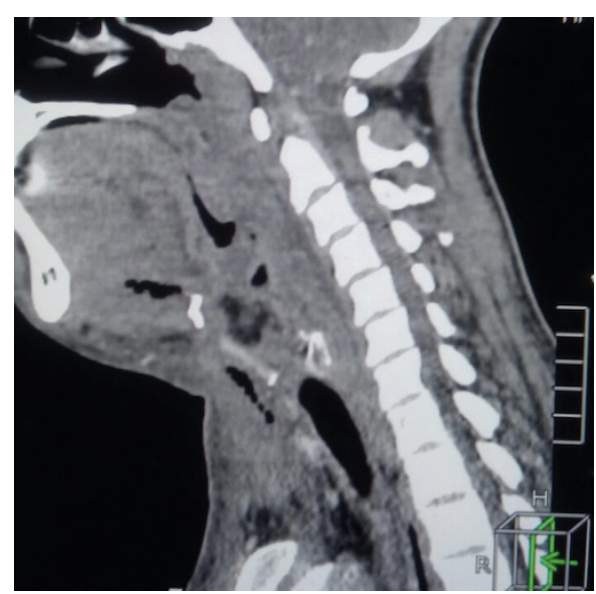

Figure 1. Computed tomography of the neck and thorax in sagittal view showing a purulent collection in the retropharyngeal space and on the deep neck space, compromising the airway.

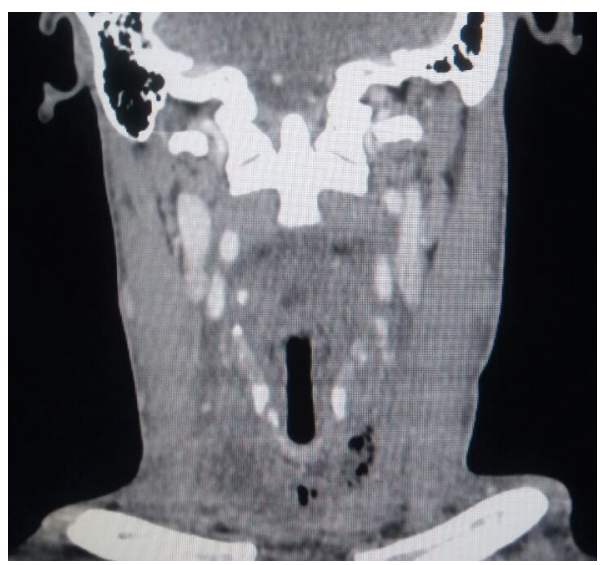

Figure 2. Computed tomography of the neck and thorax in coronal view showing a purulent collection medial do the sternocleidomastoid muscle.

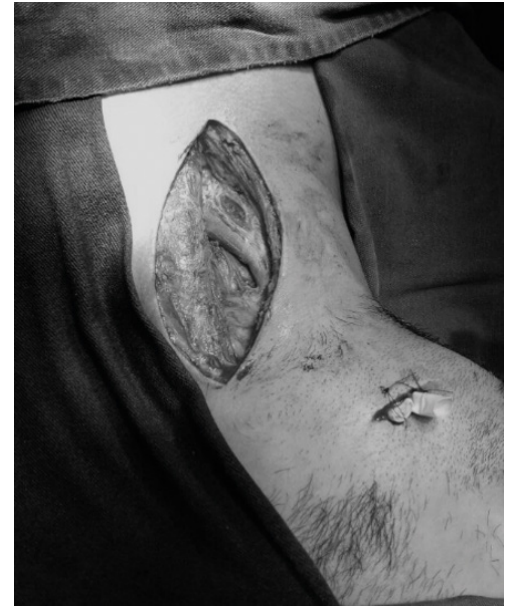

Figure 3. Transoperatory view showing the surgical wound and the poor aspect of the fascia, that appears with a pale color. 
an abundance of gram-positive cocci, which lead us to withdraw gentamicin from the scheme because it was not effective in this situation. Them, the antibiotics were changed to ceftriaxone and clindamycin. After this approach, the patient had a significant and progressive improvement of the condition, with regression of erythema and cervical edema, improvement of dyspnea and dysphagia. Pre-discharge laboratory tests showed normalization of laboratory tests. The patient did not show impairment of renal function or hydroelectrolytic imbalance. He remained hospitalized for 14 days, feeding through a nasoenteral tube, breathing in ambient air without effort. The widest cervical wound was covered daily with special dressings for 14 days, with complete closure. The patient remains well and had no other similar episodes.

\section{Acknowledgements}

The authors deny any conflict of interests.

\section{REFERENCES}

1. Ogle OE. Odontogenic infections. Dent Clin North Am. 2017 Apr;61(2):235-252. doi: 10.1016/j.cden.2016.11.004

2. Brito TP, Hazboun IM, Fernandes FL, Bento LR, Zappelini CEM, Chone CT, et al. Deep neck abscesso: study of 101 cases. Braz J Otorhinolaryngol. 2017 May - Jun;83(3):341-348. doi: 10.1016/j.bjorl.2016.04.004

3. Huang TT, Liu TC, Chen PR, Tseng FY, Yeh TH, Chen YS. Deep neck infection: analysis of 185 cases. Head Neck. 2004 Oct;26(10):854-60. 